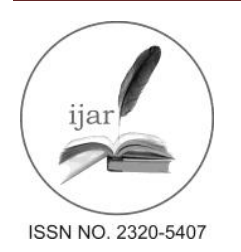

\section{Journal homepage:http://www.journalijar.com \\ Journal DOI:10.21474/IJAR01}

\section{RESEARCH ARTICLE}

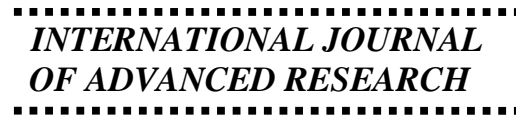

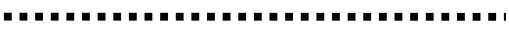

\title{
RELATIONSHIP OF DEPRESSION AND LONELINESS INDEPRESSED AND NORMAL POPULATION.
}

Fatima khurram Bukhari ${ }^{1}$, $\operatorname{IramAwan}^{2}$, Samar Fahd $^{3}$.

1. Assistant Professor, Department of Applied Psychology, The Islamia University of Bahawalpur.

2. Assistant Professor, Department of Applied Psychology, Bahaudin Zakariya University, Multan.

3. Lecturer, Department of Applied Psychology, The Islamia University of Bahawalpur.

\section{Manuscript Info}

Manuscript History:

Received: 19 March 2016

Final Accepted: 22 April 2016

Published Online: May 2016

Key words:

Depression, loneliness, population, Social Skills,

*Corresponding Author

Fatima khurram Bukhari.

\begin{abstract}
The present study was designed to explore the relationship among depression and Loneliness. The sample was consisted on 120 individuals, 60 from normal population and 60 from clinically diagnosed depressed patients. The normal sample i.e.60 individuals were further divided into 30 males' and30 females. Similarly in the sample of clinically diagnosed depressed patients 30 were males and 30 were females. Depressed patients were selected from Bahawal Victoria Hospital, Bahawalpur and normal populations were selected from the different areas of Bahawalpur. Beck's depression inventory was used to measure the level of depression and UCLA loneliness was used to measure loneliness. Result of the study reveals that there is a strong relationship among depression and loneliness. People who are depressed have strong feelings of loneliness. Which shows that people who are less assertive and lack social skills are more prone to depression which further leads to have loneliness feelings?
\end{abstract}

Copy.Right.IJAR 2016. All rights reseryed.

\section{Introduction:-}

Depression is an enthusiastic state set apart by incredible pity feelings of uselessness and Guilt, withdrawal from other and loss of rest, voracity sexual longing and hobby and joy in regular exercises. Just for instance most of us experience unhappiness throughout the way of our lives though may be not to a degree or with a frequency that warrants the diagnosis of depression is frequently associated with other psychological problems. For instance pain attacks substance abuse, sexual dysfunction and personality disorders. (Davison \& Neale, 2001).

Depression in our day by day life incorporates negative sentiments, for example, shortcoming dissatisfaction disappointment, despair helplessness and sadness. It is a little depressed state in which existence appears miserable and its contests irresistible. Clinical depression is a grave mental disorder with no positive characterization. The mental discomfort it passes is unadorned and long lasting and way intensify as the month and year go by. It is so devastating that clinically depressed individual might reach a point where they are incapable to do the simplest of life's activities and some even try to end their lives. (Davison \& Neale, 2001).

Depression belongs to the class of affective disorder in DSM-V (American Psychiatric Association, 2013). Depressed individuals face different mood changes thus it is also included in the class of mood disorders (American Psychiatric Association, 2013). At the point when depressed people are stood up to with an issue they don't appear to discover any answer for that issue. They discover each minute substantial and their heads fill and resonate with self-recrimination. Depressed individuals might likewise disregard individual cleanliness and appearance and make various hypochondriacally grievances of a throbbing painfulness that have no physical premise. They feel completely sad and lose trust and no ability to start any work. They might feel misgiving uneasiness and melancholy a significant part of the time when depression gets to be interminable, the patient does not generally snap back a prior level of working. (Davison and Neale, 2001). 
Depressed people report physical as well as psychological problems. In physical symptoms people who feel depressed might lead to weight gain or weight loss. They have broken sleep by wakeful restless spell, headaches, backaches and pain in neck and face. It is often accompanied by some physical ailments such as, indigestion, constipation, dizzy spells, unpleasant sensation in the chest and generalized pain. Psychological manifestation of depression people describes themselves as feeling apathetic, discouraged and hopeless in emotional aspects. Their expectancy for a happy and cheerful event in the future in very low and them always describe themselves in negative terms (Gara, 1995). Many depressed people feel intensely sad and dejected. They describe themselves as feeling miserable, empty and humiliated. Some of them also experience anxiety, anger or agitation. Many seem to loss their feeling of affection for friends and relatives (Comer, 1995).

Womenfolk are supplementary susceptible to have depression for the reason that the effects of hormones. Tennov found that all forms of depression are more frequently found in women than man. Women are at high risk of depression because of biological difference. Social reason may also contribute to depression among women because of larger pressures of work and family responsibility. The roles and expectations of women and increased rates of sexual abuse and poverty are the major cause of high ratio of depression in females. (Kessler,2003).

If depression is common cold of psychotherapy than loneliness is the headache (Myers, 2000). The state of loneliness, unlike solitude is a chronic distressful mental condition whereby an individual feels estranged from peers and starved from the emotional intimacy found in relationship. Aloneness is simply being away from others. Loneliness is an enthusiastic state in which an individual encounters an effective sentiment void and disconnection loneliness is more than the sentiment needing organization or needing to accomplish something with someone else.

Loneliness constitutes a ruinous type of self-recognition. The lonely get a handle on left, overlooked, unneeded and disregarded. It appears to be likely that considerations concerning the loss of past and those in it and the high see for others as appeared by counter-intuitive convictions force irrational requests on the person. Such requests ordinarily go unfulfilled making people feel more secluded in view of them and to decipher their current condition as calamitous. (Jackson2000).

Loneliness is a state of psychological discomfort which occurs when one's social relationship are smaller or less satisfactory than a person desires. The psychological discomfort are felt when our social relations lack some essential feature. This deficit may be qualitative so (too few relationships) or quantitative (unsatisfying relationships) (Sarson \& Sarson, 2000). Loneliness is neither depression nor grief. Though depression may result from loneliness. The two are not identical lonely persons are compelled to create new relationship to eliminate the stressful state they endure. Depressed individuals nevertheless are reluctant to execute their grief on others and consequently remain in separation (Schultz \& Moore, 1990).

Loneliness seems to become predominantly dominant in current periods. Toward the start of the most recent century families were commonly bigger more steady, separation was uncommon and generally few individuals lived alone in 1995, 24 million America lived in single individual family units by 2010, it is evaluated that number will have expanded to around 31 million. Physical division regularly debilitates families' securities, and these days it is not under any condition bizarre for relatives to be isolated by hundred or even a great many miles. Loneliness is often associated with negative states such as depression, anxiety and self-destructive behaviors (Rokach, 1996).

Females might be more adept to recognize their loneliness than man in light of the fact that the negative outcomes of conceding loneliness are less for females. Loneliness appears to bring down our self-esteem and low self-esteem appears to add to depression. It can be an endless loop and both add to depression. Loneliness has been observed to be identified with segregation structure kinfolk and non-family, low training low salary, passing of huge others, and unemployment, being unmarried, it is identified with negative state of mind, wrong attributions and social abilities insufficiency.

\section{Rationale of the study:-}

The present research is done to explore what relationship depression and loneliness. Loneliness has been found to relate with locus of control relational style, social network relations, interpersonal skills, social support, risk taking anxiety, depression and self-critics as well as to demographic variables. The main reason to conduct this study was to see the individual opinion about locus of control in their lives. How people relate their behavior to locus of control and how it affects their outcomes and their psychological mindedness. 
Another reason was that in Pakistani culture people know very little about loneliness. Pakistani society is based on extended family systems so there is a collective culture it may be possible that people might feel less lonely and have internal locus of control. The third and most important reason was that such type of research was not conducted in the context of Pakistani culture. The many of the researches were grounded on western culture so it is very necessary that these types of researches might be done in the Pakistani context.

Although one study was carried out in India by Dewan and Jaswal(1997) to find the relationship of depression \&locus of control in this research loneliness is not found while it is proved by many previous researches that it has an important role in depression, but Indian culture is also different from Pakistani culture and the study was on depression and locus of control but in this research another relating factor is added which might have different results. The results of research in India showed positive relation between external locus of control and depression. One of the studies was also conducted by Ayesha (2003) in order to find out the relation between depression and locus of control but loneliness is missing which is also an important factor. Her research also shows the relationship among these factors.

One of the most important purposes of this study was in this locality in the lower Punjab this topic was new and no research was conducted in this area. So the purpose was to know that the relationship either exist or not, it is a small effort to explore these dimensions which surely will help clinical psychologists, psychiatrists, and mental health professionals. It will help them to understand the cognitive styles and emotional states of the patients.

\section{Method:-}

The sample for this research was taken from Bahawalpur City one hundred and twenty individuals were taken, 60 from normal population and 60 from clinically depressed patients. These groups were further divided on the basis of gender differences and marital status. The age range was from 20-50 years having at least proper comprehension and understanding of Urdu language. Clinically depressed were taken from Bahawal Victoria Hospital Bahawalpur. The demographic variables used were age, marital status, education, occupation, \& socioeconomic class.

\section{Instruments:-}

The present study is done by the use of BDI- Urdu version (BDI-U) (Khan, 1996) to measure depression. The Beck depression inventory is a 21 item self-report questionnaire that assesses the presence and severity of cognitive, affective, motivational and physical symptoms of depression. Score range is form 0-63 higher score indicates greater symptoms severity.

The second scale was loneliness scale it was used to measure loneliness score of the individual. Every individual were rated accordingly on the basis of scoring given. Russell and Colleagues (1980) developed a revised version of UCLA (University of California Los Angelis) Loneliness scale that included positively worded or non-lonely items and negatively worded or lonely items.

\section{Procedure:-}

The sample was consisted on 120 individuals. First of all permission from administration of hospitals was granted to conduct the study. Before starting the study, informed consent of all the participants was obtained. They were assured that their information would be kept confidential and the data will be used only for research purpose. The first scale was Beck depression inventory BDI- Urdu version and the second scale was UCLA (University of California Los Angelis) Loneliness scale. When the Performa's were filled by participants, each Performa was then checked individually and rated according to the scoring manual.

\section{Result:-}

1. Multiple regressions were calculated to see the relationship between depression and loneliness.

2. Multiple regressions were used to see the relationship between depression and education.

Table No1: Correlation matrix between BDI Score and Loneliness Score LONE .

\begin{tabular}{|c|c|c|}
\hline & BDI & LONE \\
\hline DBI & - & 0.405 \\
\hline$* * \mathrm{p}<0.05$ & - & $.000 * *$ \\
\hline
\end{tabular}


Results presented in the table indicate that there is a significant correlation between the two measures. The table suggests that the correlation of BDI and LONE is $\mathrm{r}=.000(* * \mathrm{P}<.05)$. So, regarding the results depression is highly positively correlated with loneliness.

Table No 2: Model summary of the relationship between BDI Score and LONE Score.

\begin{tabular}{|c|c|c|c|c|}
\hline Model & R & R2 & Adjusted 2 & SEE \\
\hline 1 & .405 & .164 & .157 & 15.60 \\
\hline
\end{tabular}

The table indicates the model summary of the relationship between BDI and LONE. The value of R2 shows the variation in the BDI explained $16.4 \%$ variation in the BDI score is explained by the variation in the loneliness score.

Table No 3: Co-efficient of multiple regressions of BDI and Lone.

\begin{tabular}{|c|c|c|c|c|c|}
\hline \multicolumn{3}{|c|}{ Un standardized Co-efficient } & \multicolumn{3}{c|}{ Standardized Co efficient } \\
\hline Model & B & S. E & Beta & t & Sig \\
\hline Constant & -11.13 & 7.562 & -- & -1.473 & .143 \\
\hline Lone & 0.677 & 0.141 & .405 & 4.808 & .00 \\
\hline
\end{tabular}

The regression co-efficient of loneliness is 0.677. It means that one degree increase in the loneliness will lead to 6.77 degree increase in BDI score of that independent variable. So, the relationship between these two variables is highly strong and they are positively correlated to each other.

Table No 4:Correlation matrix of education (edu) and BDI score.

\begin{tabular}{|c|c|c|}
\hline & BDI & EDU \\
\hline BDI & - & -0.375 \\
\hline Sig & - & $.000^{* *}$ \\
\hline
\end{tabular}

$(* * \mathrm{P}<.05)$

Results presented in the table indicate that there is a significant negative correlation between the two measures. The table suggest that the correlation of BDI score and Education table (edu) is $r=.000(* * \mathrm{P}<.05)$. So regarding the results depression is negatively correlated with education as education increases depression decreases.

Table No 5: Model summary of the relationship between BDI scores and Education.

\begin{tabular}{|c|c|c|c|c|}
\hline Model & R & R2 & Adjusted 2 & SEE \\
\hline 1 & .375 & .140 & .133 & 15.82 \\
\hline
\end{tabular}

The table shows the model summary of the relationship between BDI and education. Result in the table indicates that the value of R2 shows the variation in BDI explained by the variation in the independent variable i-e education.

Table No 6: Co-efficient of multiple regressions of BDI and education

\begin{tabular}{|c|c|c|c|c|c|}
\hline \multicolumn{3}{|c|}{ Un standardized Co-efficient } & \multicolumn{3}{c|}{ Standardized Co efficient } \\
\hline Model & B & S. E & Beta & t & Sig \\
\hline Constant & 44.300 & 4.721 & -- & 9.384 & .000 \\
\hline EDU & -5.818 & 1.325 & -.375 & -4.391 & .000 \\
\hline
\end{tabular}

Results in the table show the regression coefficient of education (edu) which is -5.232. It means that one degree increase in the education tends then it would lead to -5.818 degree decrease in the BDI score of that independent variable. So, the negative correlation exists between these two variables.

\section{Discussion:-}

Every person has a wish to live a life in which he gets all the luxuries of life. It is a mechanical world today. Everyone has to work as a machine to get the luxuries of life. Everyone is running towards comfort. So there is a competition between people. Everyone is sacrificing his/her peace of mind to get all comfort. So, this type of environment causes many problems which arises because of the failure is depression. People who do not cope with this fast world may become depressed.People sometimes feeling depressed are also left the societies they lost their confidence in themselves and then they remain apart from all social gatherings, so, a sense of loneliness developed. Those people preferred to live a lonely life. They don't have friends. They make their own fantasies and live in those fantasies. Their psychological awareness is very poor. In our society people don't have any concept about the psychological disorders. So, the unawareness of the psychological disorder plays an important role in the development if these disorders. 
Present study was assumed to investigate relationship of depression and loneliness among normal and depressed individuals. The research was conducted on the basis of two different scales. For the measurement of depression, Beck depression inventory was used. In contrast for the measurement of loneliness UCLA loneliness scale was used to measure loneliness. The main aim of the study was to see the relationship between depression and loneliness.

The results show that a very strong relationship exist between these two variables. So, depressed people also feel lonely and they developed loneliness. According to Rubinstein and Shaver loneliness seem to lower self-esteem and contribute to depression. It can be a vicious circle and both contribute to loneliness. According to researches it is found that person who exhibited cognitive strategy called pessimistic avoidance (Characterized by failure expectations negative effects, avoidance and self-handicapping) was related to loneliness that persisted over a period of one year. This finding has support from other researches, which show that lonely person has a general pessimistic attitude towards social situations (Parkhust\&Hopmeyer, 1999). This pessimistic attitude in parts prevent lonely individuals from forming new attachments with other for fear of rejection perceived as inevitable because of a possible combination between expectations of failure and feelings of social inadequacy.

Related to the general concept of social skills deficits is the somewhat more specific idea that constant seeking a reassurance is the critical variable in depression. Depressed people seek reassurance that other truly care but even when reassured they are only temporarily satisfied. Their negative self- concept causes them to double the truth of the feedback they have received. So, these people leave the society and they developed feelings of loneliness (Davison \& Neale, 2001). Another objective was to see relation of education and depression. Result suggests that education is negative correlated to depression. Education plays important role as education increases then depression decreases, because it gives awareness of the disturbance and a can better cope with the depression.

All these play important role in the etiology of depression. Some social factors such as family environment and people attitude. The behavior of the people stress others to become pessimistic they lack self-confidence because of the negative attitude of people.

\section{Conclusion:-}

This research reveals that the relationship exists between depression and loneliness. It is concluded that because of the stress in life people became disappointed and they feel lonely and separate themselves from their environment. It is concluded form the research that these variables are interrelated and occurrence of one of the variable leads to the cause of other.

\section{References:-}

1. American Psychiatric Association. (2013). Diagnostic and Statistical Manual of Mental Disorders (DSM-5). American Psychiatric Pub.

2. Comer R.J. (1995).Abnormal Psychology ( $2^{\text {nd }}$ Ed.) USA: W.H. Freeman \&Company.

3. Davison G.C \& Neale, J.M. (2001).Abnormal Psychology ( $8^{\text {th }}$ Ed.). New York:John Wiley \& Sons.

4. Jackson, K. (2000). Family Homelessness: More than Simply a Lack of Housing. Taylor \& Francis.

5. Jaswal, S., \&Dewan, A. (1997).The Relationship between Locus of Control and Depression. Journal of Personality and Clinical Studies, 13, 25-28.

6. Kessler, R. C. (2003). Epidemiology of Women and Depression. Journal of Affective Disorders, 74(1), 5-13.

7. Khan, A. A., Marwat, S. K., Noor, M. M., \& Fatima, S. (2015). Reliability and Validity of Beck Depression Inventory among General Population in Khyber Pakhtunkhwa, Pakistan. Journal of Ayub Medical College Abbottabad, 27(3), 573-575.

8. Myers, D. G. (2000). The Funds, Friends, and Faith of Happy People. American Psychologist, $55(1), 56$.

9. Parkhurst, J. T., \&Hopmeyer, A. (1999). Developmental Change in the Sources of Loneliness in Childhood and Adolescence: Constructing a Theoretical Model. Loneliness in Childhood and Adolescence, 56-79.

10. Rokach, A., \& Brock, H. (1996). The Causes of Loneliness. Psychology: A Journal of Human Behavior.

11. Samreen, H., \&Zubair, A. (2013).Locus of Control and Death Anxiety among Police Personnel. Pakistan Journal of Psychological Research, 28(2), 261.

12. Sarson, GJ. \&Sarson, R.B. (2000).Abnormal Psychology $\left(6^{\text {th }}\right.$ Ed). Prentice Hall, New Jersey.

13. Schultz, N. R., \& Moore, D. (1986). The Loneliness Experience of College Students Sex Differences. Personality and Social Psychology Bulletin,12(1), 111-119.

14. Woolfolk, R. L., Novalany, J., Gara, M. A., Allen, L. A., \&Polino, M. (1995). Self-Complexity, Self-Evaluation, and Depression: An Examination of Form and Content within the Self-Schema. Journal of Personality and Social Psychology, 68(6), 1108. 